\title{
Treadmill exercise ameliorates depressive symptoms through increasing serotonin expression in postpartum depression rats
}

\author{
Eun-Sang Ji', Jae-Min Lee', Tae-Woon Kim¹, You-Mi Kim² , Yeon-Soo Kim², Kijeong Kim,* \\ 'Department of Physiology, College of Medicine, Kyung Hee University, Seoul, Korea \\ ${ }^{2}$ Department of Sport and Leisure Studies, Seonam University, Asan, Korea \\ ${ }^{3}$ School of Exercise \& Sport Science, College of Natural Sciences, University of Ulsan, Ulsan, Korea
}

Postpartum depression (PPD) is defined as the depressive symptoms that occur from the moment of delivery until 12 months after delivery. PPD symptoms are closely associated with reduced activity of the serotonergic system. Serotonin (5-hydroxytryptamine, 5-HT) plays an important role in the pathogenesis of depression. Tryptophan hydroxylase (TPH) catalyzes the rate-limiting step of 5-HT biosynthesis in the serotonergic neurons. Exercise exerts anti-depressive effect on depression patients as well as on animal models of depression. In the present study, the effect of treadmill exercise on PPD was investigated using rats. For this study, open field test for activity and forced swimming test for depressive symptoms, and immunohistochemistry for 5-HT and TPH were conducted. The rats in the exercise groups were forced to run on a motorized treadmill for 30 min once a day for 2 weeks. Activity in the open field test was decreased in the postpartum rats, however, performing treadmill running increased activity in the postpartum rats. The climbing time was decreased and the immobility time was increased in the postpartum rats. Treadmill exercise increased climbing time and suppressed immobility time in the postpartum rats. 5-HT and TPH expressions in the dorsal raphe were suppressed in the postpartum rats, and treadmill exercise enhanced 5-HT and TPH expressions in the postpartum rats. Treadmill exercise ameliorated the PPD very effectively by enhancing serotonin level.

Keywords: Postpartum depression, 5-Hydroxytryptamine, Tryptophan hydroxylase, Treadmill exercise

\section{INTRODUCTION}

Postpartum depression (PPD) is defined as the depressive symptoms that occur from the moment of delivery until 12 months after delivery (Gaynes et al., 2005). Mild depressive symptoms are followed after delivery, and approximately $50 \%$ of women experience 'postpartum blues' (Fernandez et al., 2014). Postpartum blues occur in 15\%-85\% of women within the first 10 days after delivery (Henshaw, 2003), and it is also a psychiatric disorder that occurs in 10\%-15\% of childbearing women (Arbabi et al., 2014). Although postpartum blues is a common and transient occurrence and generally does not require intervention, its recognition is important because postpartum blues is a risk factor inducing subsequent PPD (Reck et al., 2009). PPD is defined strictly in the psychiatric nomenclature as a major depressive disorder (Pearlstein et al., 2009).

The symptoms of PPD are closely associated with reduced activity of the serotonergic system (Baek et al., 2012). Serotonin, also known as 5-hydroxytryptamine (5-HT), is a monoamine neurotransmitter, which acts as a biochemical messenger and regulator in the brain functions (Kim et al., 2015). 5-HT is closely associated with depression, and decrement in 5-HT metabolism was ascertained in the brains of a subgroup of patients with depression (Baek et al., 2012). Tryptophan hydroxylase (TPH) catalyzes the rate-limiting step of 5-HT biosynthesis in the serotonergic neurons, therefore 5-HT and TPH expressions are related with the severity of de-
${ }^{*}$ Corresponding author: Kijeong Kim (ib http://orcid.org/0000-0001-9233-3096 School of Exercise \& Sport Science, College of Natural Sciences, University of Ulsan, 93 Daehak-ro, Nam-gu, Ulsan 44610, Korea

Tel: +82-52-259-2381, Fax: +82-52-259-1696, E-mail: kijeongk@ulsan.ac.kr Received: February 1, 2017 / Accepted: April 19, 2017
This is an Open Access article distributed under the terms of the Creative Commons Attribution Non-Commercial License (http://creativecommons.org/licenses/by-nc/4.0/) which permits unrestricted non-commercial use, distribution, and reproduction in any medium, provided the original work is properly cited. 
pression (Roh et al., 2016). In many animal experiments and human studies, serotonin expression level is the cause of behavioral and mental illness (Gainetdinov et al., 1999; Murphy et al., 2003).

Exercise exerts anti-depressive effect on depression patients as well as on animal models of depression (Baek et al., 2012; Blumenthal et al., 1999; Kim et al., 2015). In the present study, we investigated the effect of treadmill exercise on PPD using rats. For this study, open field test for activity and forced swimming test for depressive symptoms, and immunohistochemistry for 5-HT and TPH were conducted.

\section{MATERIALS AND METHODS}

\section{Animals and treatments}

The experimental procedures were performed in accordance with the animal care guidelines of the National Institutes of Health and the Korean Academy of Medical Sciences. The animals were housed under controlled temperature $\left(23^{\circ} \mathrm{C} \pm 2^{\circ} \mathrm{C}\right)$ and lighting (08:00 a.m. to 20:00 p.m.) conditions with food and water available ad libitum. The pregnant Sprague-Dawley rats (10 weeks old) were obtained from commercial breeder (Orient Co., Seoul, Korea), and randomly divided into 4 groups ( $\mathrm{n}=8$ in each group): the control group, the control and exercise group, the PPD group, and the PPD and exercise group.

\section{Treadmill exercise protocol}

After delivery, the rats in the exercise groups were forced to run on a motorized treadmill for 30 min once a day for 2 weeks. Exercise load for the running group consisted of running at a speed of 2 $\mathrm{m} / \mathrm{min}$ for the first $5 \mathrm{~min}$, at a speed of $5 \mathrm{~m} / \mathrm{min}$ for the next $5 \mathrm{~min}$, and then at a speed of $8 \mathrm{~m} / \mathrm{min}$ for the last $20 \mathrm{~min}$, with the $0^{\circ}$ inclination. The rats in the control groups were left on the treadmill without running for the same period as the exercise groups.

\section{Open field test}

Activity was determined using the open field test according to the previously described method (Roh et al., 2016). The animals were randomly assigned to an order of testing and placed in a white square open field arena $(100 \mathrm{~cm} \times 100 \mathrm{~cm})$ made of wood. It was enclosed with 40-cm-high walls and placed under strong illumination (200 lux). The arena was divided into 25 squares (each square is $20 \mathrm{~cm} \times 20 \mathrm{~cm}$ ), defined as 9 central and 16 peripheral squares. The animal was placed in the center of the arena and left free to explore the environment for $1 \mathrm{~min}$. After that time, the numbers of squares that the rat crossed were recorded for $5 \mathrm{~min}$.

\section{Forced swimming test}

Forced swimming test was conducted according to the previously described method (Kim et al., 2015; Roh et al., 2016). Experimental animals were dropped individually into glass cylinders. The glass cylinder (height, $50 \mathrm{~cm}$; diameter, $15 \mathrm{~cm}$ ) contained water at a temperature of $27^{\circ} \mathrm{C}$. The water depth was 30 $\mathrm{cm}$. All of the experimental animals underwent a pretest for 15 min to eliminate the acute stress of the water and to provide the animals with the ability to adapt to the water. Twenty-four hours after pretest, the animals were tested for 5 min. During the test session, the climbing time and the immobility time were analyzed using a Smart version 2.5 video tracking system (Panlab, Barcelona, Spain). Immobility behavior was defined to occur when no additional activity was observed other than the actions needed to keep the animals' head above the water. Climbing behavior consisted of upward-directed movements of the forepaws along the side of the swim chamber.

\section{Tissue preparation}

The animals were sacrificed immediately after performing the forced swimming test. To begin the sacrifice process, the animals were fully anesthetized using Zoletil $50(10 \mathrm{mg} / \mathrm{kg}$, intraperitoneally; Vibac Laboratories, Carros, France). The anesthetized rats were transcardially perfused with $50-\mathrm{mM}$ phosphate-buffered saline (PBS), and fixed with a freshly prepared solution consisting of $4 \%$ paraformaldehyde in 100-mM phosphate buffer at $\mathrm{pH} 7.4$. Brains were dissected, post-fixed in the same fixative overnight, and transferred to $30 \%$ sucrose for cryoprotection. Coronal sections of $40-\mu \mathrm{m}$ thickness were made with a freezing microtome (Leica, Nussloch, Germany). The sections were finally mounted onto gelatin-coated slides. The slides were air-dried overnight at room temperature, and the coverslips were mounted using Permount (Fisher Scientific Inc., Waltham, MA, USA).

\section{5-HT and TPH Immunohistochemistry}

Immunohistochemistry for 5-HT and TPH was performed according to the previously described method (Kim et al., 2015; Roh et al., 2016). Briefly, the sections were incubated in PBS for 10 min and washed 3 times, again with PBS and then incubated in $1 \% \mathrm{H}_{2} \mathrm{O}_{2}$ for $30 \mathrm{~min}$. Next, the sections were incubated overnight with rabbit anti-5-HT antibody (Immunostar, Hudson, NY, USA) at a dilution of 1:500 for visualization of 5-HT expression or with mouse anti-TPH antibody (Oncogene Research Product, Cambridge, UK) at a dilution of 1:1,000 for visualization of TPH expression. The sections were then incubated for $1 \mathrm{hr}$ 


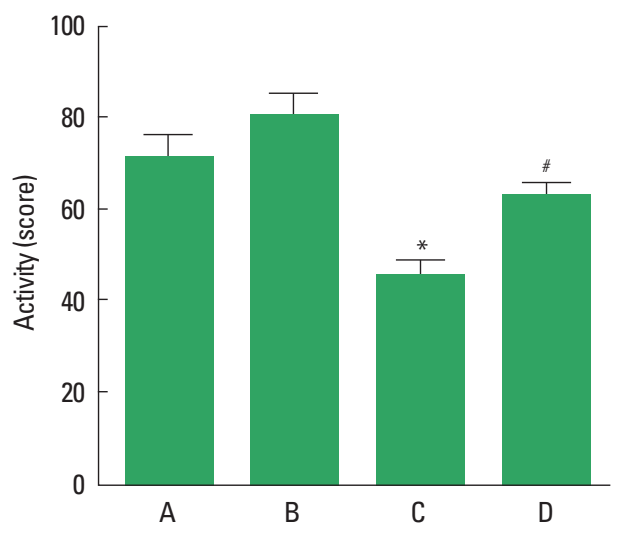

Fig. 1. Effect of treadmill exercise on the activity in the open field test. A, control group; B, control and exercise group; $C$, postpartum depression group; D, postpartum depression and exercise group. ${ }^{*} P<0.05$ compared to the control group. ${ }^{\#} P<0.05$ compared to the postpartum depression group. The data are expressed as the mean \pm standard error of the mean.

with biotinylated anti-rabbit secondary antibody or with anti-mouse secondary antibody (Vector Laboratories, Burlingame, CA, USA). The sections were subsequently incubated with avidin-biotin-peroxidase complex (Vector Laboratories) for $1 \mathrm{hr}$ at room temperature. Immunoreactivity was visualized by incubating the sections in a solution consisting of $0.05 \% 3,3^{\prime}$-diaminobenzidine and $0.01 \% \mathrm{H}_{2} \mathrm{O}_{2}$ in $50-\mathrm{mM}$ Tris-buffer $(\mathrm{pH}, 7.6)$ for approximately $3 \mathrm{~min}$. The sections were then mounted on gelatin-coated glass slides. The slides were air-dried overnight at room temperature and dehydrated through a gradient of ethanol and covered with coverslips using Permount (Fisher Scientific Inc.).

\section{Data analysis}

The numbers of 5-HT-positive and TPH-positive cells in the dorsal raphe were counted hemilaterally under a light microscope (Olympus, Tokyo, Japan), and they were expressed as the numbers of cells/section of the dorsal raphe. Statistical analysis was performed using one-way analysis of variance followed by Duncan's post-boc test. The results are presented as the mean \pm standard error of the mean. Significance was set as $P<0.05$.

\section{RESULTS}

\section{Effect of treadmill exercise on the activity in the open field test}

The activity score of the open field test is presented in Fig. 1. The present results indicate that the activity score in the PPD group was decreased compared to the control group $(P<0.05)$.
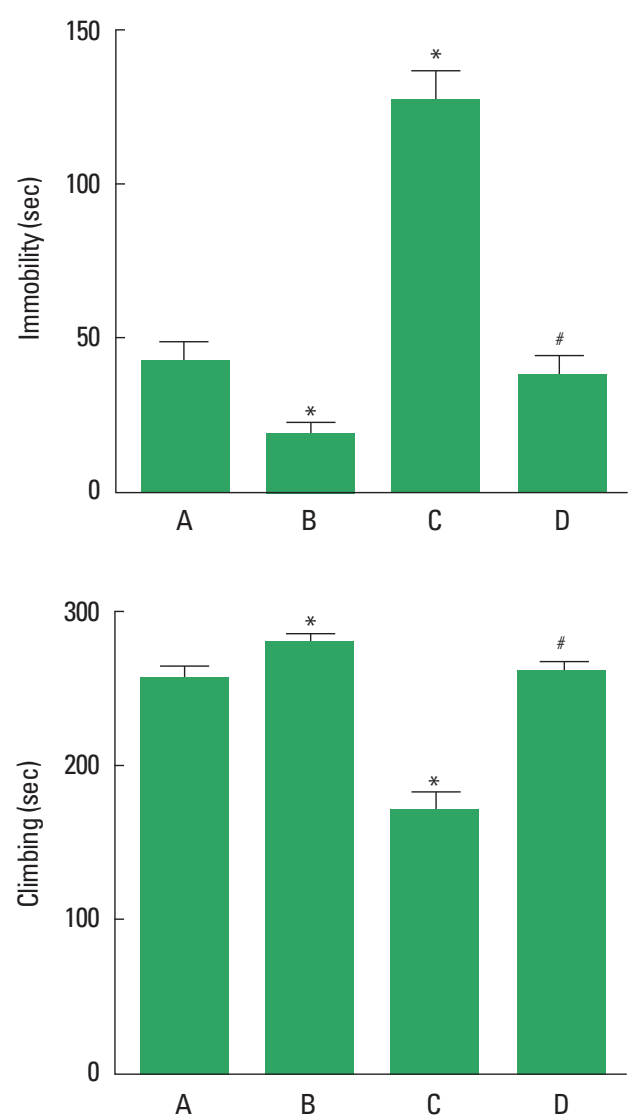

Fig. 2. Effect of treadmill exercise on the depressive behaviors in the forced swimming test. $A$, control group; $B$, control and exercise group; $C$, postpartum depression group; $\mathrm{D}$, postpartum depression and exercise group. ${ }^{*} P<0.05$ compared to the control group. ${ }^{\#} P<0.05$ compared to the postpartum depression group. The data are expressed as the mean \pm standard error of the mean.

However, treadmill exercise increased the activity in the PPD group $(P<0.05)$.

\section{Effect of treadmill exercise on the depressive behaviors in the forced swimming test}

The time of the immobility and the time of climbing in the forced swimming test are presented in Fig. 2. The present result indicate that immobility time was increased and climbing time was decreased in the PPD group compared to the control group $(P<0.05)$. However, treadmill exercise decreased the immobility time and increased the climbing time in the PPD group $(P<0.05)$.

\section{Effect of treadmill exercise on the 5-HT expression in the dorsal raphe}

The effect of treadmill exercise on the 5-HT expression in the dorsal raphe is presented in Fig. 3. The present results indicate 

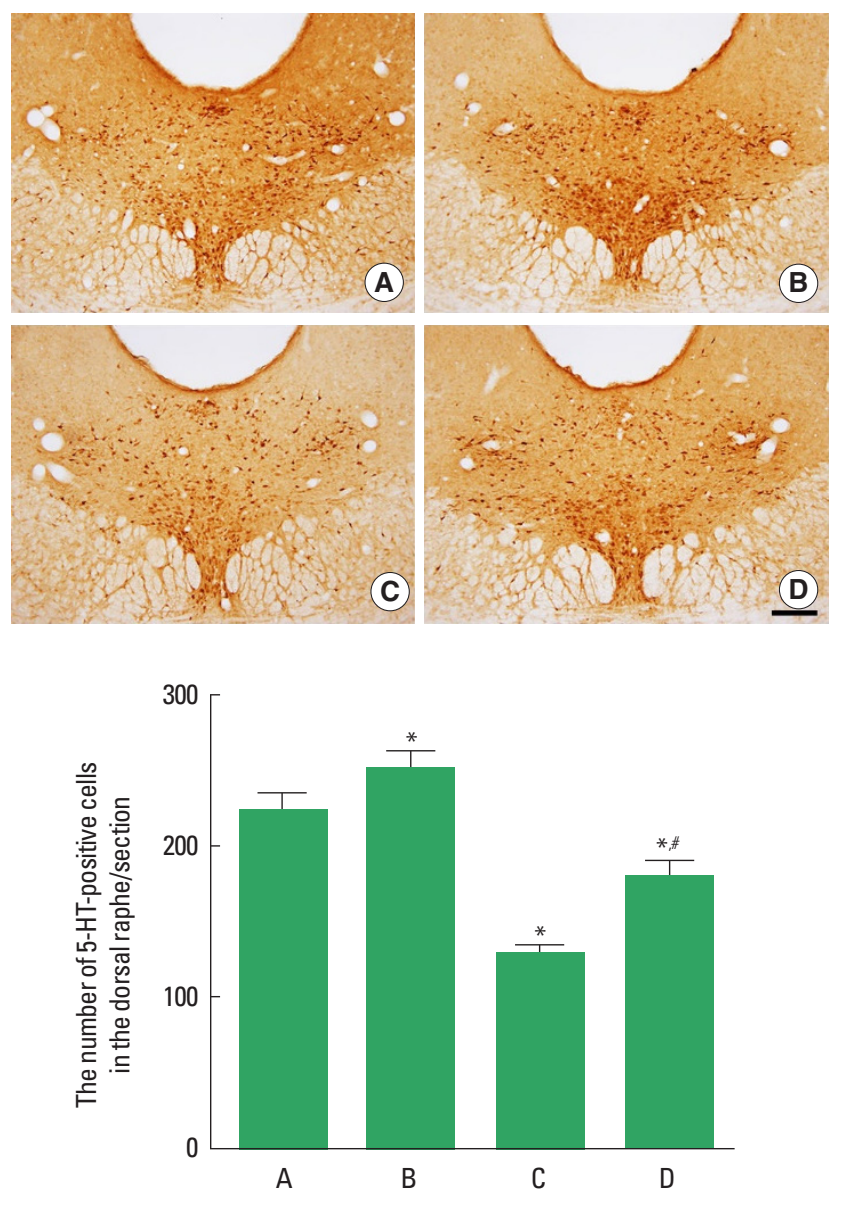

Fig. 3. Effect of treadmill exercise on the 5-hydroxytryptamine (5-HT) expression in the dorsal raphe. Upper panel: Photomicrographs of 5-HT-positive cells. The scale bar represents $200 \mu \mathrm{m}$. Lower panel: Number of 5 -HT-positive cells in each group. $A$, control group; $B$, control and exercise group; $C$, postpartum depression group; $\mathrm{D}$, postpartum depression and exercise group. ${ }^{*} P<0.05$ compared to the control group. ${ }^{*} P<0.05$ compared to the postpartum depression group. The data are expressed as the mean \pm standard error of the mean.

that the number of 5-HT-positive cells in the dorsal raphe was decreased in the PPD group compared to the control group $(P<$ $0.05)$. In contrast, treadmill exercise increased the number of 5-HT-positive cells in the PPD group $(P<0.05)$.

\section{Effect of treadmill exercise on the TPH expression in the dorsal raphe}

The effect of treadmill exercise on the TPH expression in the dorsal raphe is presented in Fig. 4. The present results indicate that the number of TPH-positive cells in the dorsal raphe was decreased in the PPD group compared to the control group $(P<$ $0.05)$. In contrast, treadmill exercise increased the number of TPH-positive cells in the PPD group $(P<0.05)$.
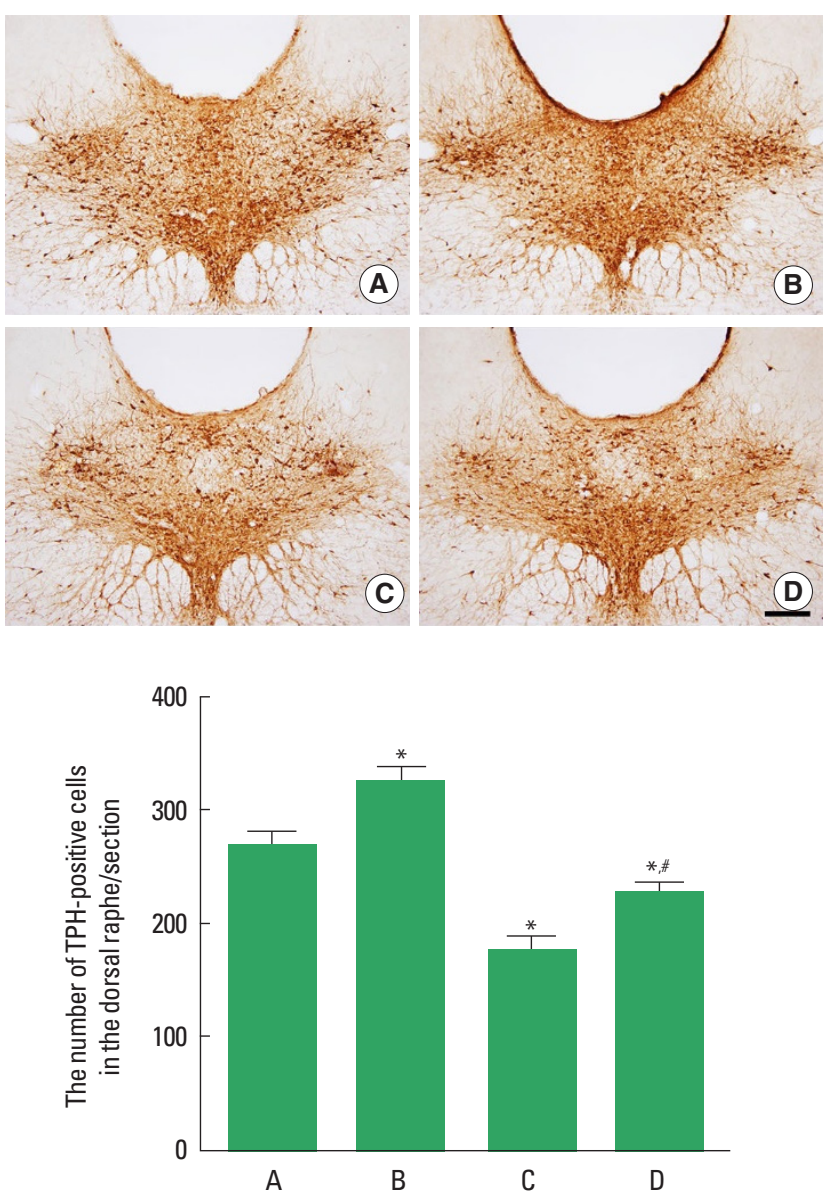

Fig. 4. Effect of treadmill exercise on the tryptophan hydroxylase (TPH) expression in the dorsal raphe. Upper panel: Photomicrographs of TPH-positive cells. The scale bar represents $200 \mu \mathrm{m}$. Lower panel: Number of TPH-positive cells in each group. $A$, control group; $B$, control and exercise group; $C$, postpartum depression group; $\mathrm{D}$, postpartum depression and exercise group. ${ }^{*} P<0.05$ compared to the control group. ${ }^{\sharp} P<0.05$ compared to the postpartum depression group. The data are expressed as the mean \pm standard error of the mean.

\section{DISCUSSION}

Exercise is effective for reducing the symptoms of depression and anxiety (Wipfli et al., 2011). Open field activity is a measure for the anxiety level (Shirayama et al., 2002). In the present study, activity in the open field test was decreased in the postpartum rats, however, performing treadmill running increased activity in the postpartum rats. The present results show that treadmill exercise alleviated postpartum-induced anxiety.

A decrease in the climbing time and an increase in the immobility time of the forced swimming test indicate depressive state (Craft et al., 2010; Kim et al., 2012). In the present study, the climbing time was decreased and the immobility time was in- 
creased in the postpartum rats. Treadmill exercise increased climbing time and suppressed immobility time in the postpartum rats. These results demonstrate that treadmill exercise exerted an anti-depressive effect on the postpartum rats.

Dysregulation of brain serotonin is an important contributing factor for many psychiatric disorders. Variations of the brain 5-HT levels contribute to behavioral alterations in mice (Gainetdinov et al., 1999) and psychiatric disorders in humans (Murphy et al., 2003). The 5-HT in the dorsal and median raphe nuclei ramifies to most of the brain regions, and 5-HT is involved in the mood regulation, and serotonergic dysfunction is closely associated with mood disorders, such as anxiety, depression, panic, and obsessive compulsive disorder (Cools et al., 2008; Kim et al., 2015). TPH is an enzyme that regulates the synthesis of the 5-HT, and is used as an indicator of serotonin synthesis. Increment of 5-HT synthesis and enhancement of TPH expression caused by treadmill exercise is well documented (Baek et al., 2012; Lee et al., 2013). Kim et al. (2015) showed that treadmill exercise increased 5-HT and TPH expressions and ameliorated depression symptoms. In the present study, 5-HT and TPH expressions in the dorsal raphe were suppressed in the postpartum rats, and treadmill exercise enhanced 5-HT and TPH expressions in the postpartum rats. These results demonstrate that treadmill exercise restored serotonin level following PPD.

In the present results, treadmill exercise ameliorated the PPD very effectively by enhancing serotonin level. Therefore these results support the theory that exercise may provide a potential therapeutic strategy for the alleviation of depression symptoms in PPD patients.

\section{CONFLICT OF INTEREST}

No potential conflict of interest relevant to this article was reported.

\section{ACKNOWLEDGMENTS}

This work was supported by the National Research Foundation of Korea Grant funded by the Korean Government (NRF2014S1A5A8015969).

\section{REFERENCES}

Arbabi L, Baharuldin MT, Moklas MA, Fakurazi S, Muhammad SI. Antidepressant-like effects of omega-3 fatty acids in postpartum model of depression in rats. Behav Brain Res 2014;271:65-71.

Baek SS, Jun TW, Kim KJ, Shin MS, Kang SY, Kim CJ. Effects of postnatal treadmill exercise on apoptotic neuronal cell death and cell proliferation of maternal-separated rat pups. Brain Dev 2012;34:45-56.

Blumenthal JA, Babyak MA, Moore KA, Craighead WE, Herman S, Khatri P, Waugh R, Napolitano MA, Forman LM, Appelbaum M, Doraiswamy PM, Krishnan KR. Effects of exercise training on older patients with major depression. Arch Intern Med 1999;159:2349-2356.

Cools R, Roberts AC, Robbins TW. Serotoninergic regulation of emotional and behavioural control processes. Trends Cogn Sci 2008;12:31-40.

Craft RM, Kostick ML, Rogers JA, White CL, Tsutsui KT. Forced swim test behavior in postpartum rats. Pharmacol Biochem Behav 2010;96: 402-412.

Fernandez JW, Grizzell JA, Philpot RM, Wecker L. Postpartum depression in rats: differences in swim test immobility, sucrose preference and nurturing behaviors. Behav Brain Res 2014;272:75-82.

Gainetdinov RR, Wetsel WC, Jones SR, Levin ED, Jaber M, Caron MG. Role of serotonin in the paradoxical calming effect of psychostimulants on hyperactivity. Science 1999;283:397-401.

Gaynes BN, Gavin N, Meltzer-Brody S, Lohr KN, Swinson T, Gartlehner G, Brody S, Miller WC. Perinatal depression: prevalence, screening accuracy, and screening outcomes. Evid Rep Technol Assess (Summ) 2005;(119):1-8.

Henshaw C. Mood disturbance in the early puerperium: a review. Arch Womens Ment Health 2003;6 Suppl 2:S33-42.

Kim JE, Ji ES, Seo JH, Lee MH, Cho S, Pak YK, Seo TB, Kim CJ. Alcohol exposure induces depression-like behavior by decreasing hippocampal neuronal proliferation through inhibition of the BDNF-ERK pathway in gerbils. Anim Cells Syst 2012;16:190-197.

Kim TW, Lim BV, Baek D, Ryu DS, Seo JH. Stress-Induced Depression Is Alleviated by Aerobic Exercise Through Up-Regulation of 5-Hydroxytryptamine 1A Receptors in Rats. Int Neurourol J 2015;19:27-33.

Lee SW, Kim YS, Jun TW, Seo JH, Kim K, Shin MS, Kim CJ. The impact of duration of one bout treadmill exercise on cell proliferation and central fatigue in rats. J Exerc Rehabil 2013;9:463-469.

Murphy DL, Uhl GR, Holmes A, Ren-Patterson R, Hall FS, Sora I, Detera-Wadleigh S, Lesch KP. Experimental gene interaction studies with SERT mutant mice as models for human polygenic and epistatic traits and disorders. Genes Brain Behav 2003;2:350-364.

Pearlstein T, Howard M, Salisbury A, Zlotnick C. Postpartum depression. Am J Obstet Gynecol 2009;200:357-364.

Reck C, Stehle E, Reinig K, Mundt C. Maternity blues as a predictor of DSM-IV depression and anxiety disorders in the first three months postpartum. J Affect Disord 2009;113:77-87.

Roh JH, Ko IG, Kim SE, Lee JM, Ji ES, Kim JH, Chang HK, Lee SK, Kim 
$\mathrm{KH}$. Treadmill exercise ameliorates intracerebral hemorrhage-induced depression in rats. J Exerc Rehabil 2016;12:299-307.

Shirayama Y, Chen AC, Nakagawa S, Russell DS, Duman RS. Brain-derived neurotrophic factor produces antidepressant effects in behavior- al models of depression. J Neurosci 2002;22:3251-3261.

Wipfli B, Landers D, Nagoshi C, Ringenbach S. An examination of serotonin and psychological variables in the relationship between exercise and mental health. Scand J Med Sci Sports 2011;21:474-481. 